See discussions, stats, and author profiles for this publication at: https://www.researchgate.net/publication/264512152

\title{
Submesoscale structures monitoring and detection by satellite imagery: El Hierro island submarine volcano
}

Conference Paper · July 2014

DOI: 10.1109/IGARSS.2014.6946948

CITATIONS

0

3 authors, including:

a

Javier Marcello-Ruiz

Universidad de Las Palmas de Gran Canaria

16 PUBLICATIONS 16 CITATIONS

SEE PROFILE

Some of the authors of this publication are also working on these related projects:

H2020 Impressive View project

Project IMPRESSIVE View project
READS

50

Javier Martin Abasolo

Universidad de Las Palmas de Gran Canaria

27 PUBLICATIONS 200 CITATIONS

SEE PROFILE 


\title{
SUBMESOSCALE STRUCTURES MONITORING AND DETECTION BY SATELLITE IMAGERY: EL HIERRO ISLAND SUBMARINE VOLCANO
}

\author{
Francisco Eugenio, Javier Marcello, Javier Martin \\ Instituto de Oceanografía y Cambio Global, Universidad de Las Palmas de Gran Canaria \\ Campus Universitario de Tafira, 35017 Las Palmas de Gran Canaria, Spain
}

\begin{abstract}
The island off the Atlantic coast of North Africa, El Hierro (Canary Island), has been rocked by thousands of tremors and earthquakes since July 2011, and an underwater volcanic eruption 300 meters below sea level started on October 10, 2011. Thanks to this natural tracer release, low resolution satellite images obtained from MODIS and MERIS sensors have been processed for monitoring the volcanic plume evolution and it has provided a unique and outstanding source of tracer that may allow us to study submesoscale front-like and filament-like structures. These structures have been monitored and detected using an advanced methodology based on an initial structure segmentation algorithm combined by a structure growing technique.
\end{abstract}

Index Terms - El Hierro submarine volcano, MODIS, MERIS, image segmentation, submesoscale structures.

\section{INTRODUCTION}

Satellite remote sensing is providing a systematic, synoptic framework for advancing scientific knowledge of the Earth as a complex system of geophysical phenomena that, directly and through interacting processes, often lead to natural hazards. The recent eruption of a submarine volcano at the El Hierro Island has provided a unique and outstanding source of tracer that may allow us to study a variety of structures. The island off the Atlantic coast of North Africa - built mostly from a shield volcano- - has been rocked by thousands of tremors and earthquakes since July 2011, and an underwater volcanic eruption 300 meters below sea level started on October 10, 2011. Thanks to this natural tracer release, low and high-resolution satellite images obtained from MODIS, MERIS and WorldView sensors have been processed to provide information on the concentration of a number of marine parameters: chlorophyll, phytoplankton, suspended matter, etc. This oceanographic remote sensing data has played, as well, a fundamental role during field campaigns guiding the Spanish government oceanographic vessel to the appropriate sampling areas [1]. In this context, this paper illustrates the capabilities of satellite remote sensing systems, specifically MODIS and MERIS images, to improve the understanding of submarine volcanic processes and hazards. Finally, it has to be pointed out that this natural tracer release has been monitored and detected using an advanced methodology based on an initial structure detection using the appropriate segmentation algorithm plus a subsequent structure growing technique to detect the submesoscale structures. This detection approach has been validated over a database of MERIS and MODIS oceanographic products and it has demonstrated an excellent performance and robustness.

\section{SATELLITE REMOTE SENSING DATA}

The last two decades have witnessed the increasing use of remote sensing for understanding the geophysical phenomena underlying natural hazards. The contributions of satellite remote sensing to Earth science, ranging from highresolution topography (using SAR, Lidar, etc.) and geodesy to passive multispectral (such as MODIS, MERIS, WorldView-2, Hyperion, etc.) and active microwave imaging, have transformed the discipline [2]. In this context, a regular multidisciplinary monitoring of the El Hierro underwater volcano has been carried out in order to quantify the environmental impact [3]. The eruption is the first in the island chain in nearly 40 years and it is just off the southern coast of El Hierro, as shown in Figure 1 (a). A milky green plume in the water stretched 25-30 kilometers at its widest and, approximately, 100 kilometers long, from a large mass near the coast to thin tendrils as it spreads to the southwest, as shown in the multisensorial color composite images presented in Figure 1 (b)-(d). This paper is not intended as a comprehensive treatment of the application of remote sensing to the selected natural hazards but rather as recognition of the contributions of satellite remote sensing to understanding underlying phenomena and providing critical information for decision support by emergency managers and the disaster response community. 


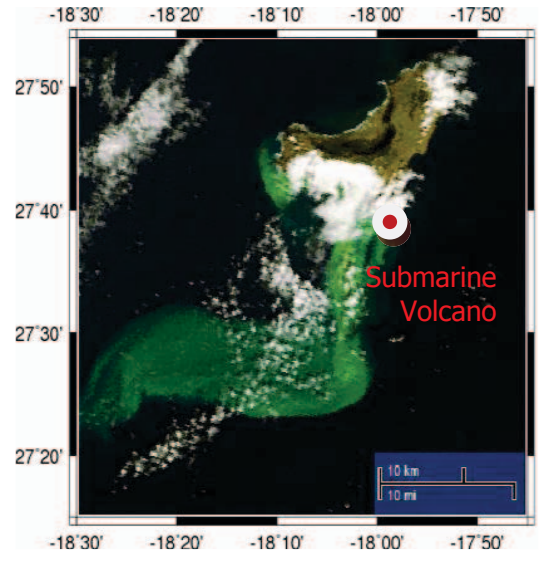

(a)

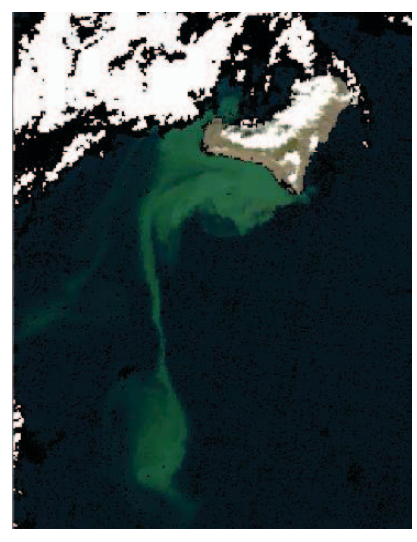

(b)

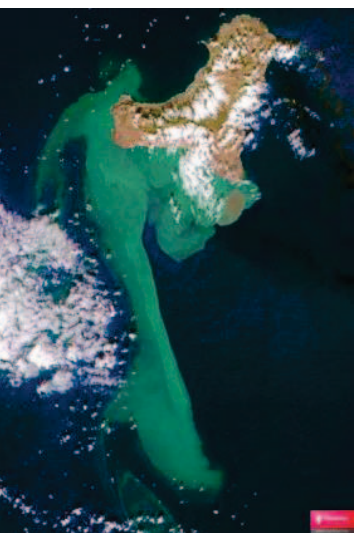

(c)

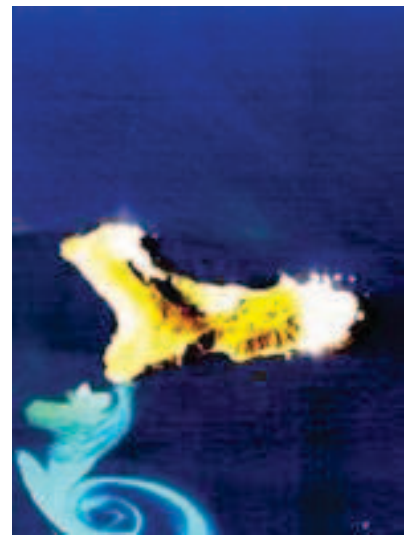

(d)

Figure 1. (a) MODIS image of El Hierro submarine volcano location $(27.78 \mathrm{~N},-18.04 \mathrm{~W})$ and, (b)-(d) multisensorial MERIS ((ESA $\left.{ }^{\mathcal{C}}\right)$ : RAPIDEYE $^{\mathcal{O}}$ and hyperspectral HYPERION remote sensing images of El Hierro volcanic plume.

\section{SUBMESOSCALE OCEANOGRAPHIC STRUCTURES: MONITORING AND DETECTION}

Satellite data from AQUA/TERRA-MODIS and ENVISATMERIS sensors have been the main source of image information to improve understanding of El Hierro submarine volcanic processes. MODIS instruments flying on both the TERRA and AQUA satellites provides excellent temporal coverage with 2 daylight and 2 night overflights per 24 hours. On the other hand, MERIS, aboard ENVISAT satellite, is a programmable, medium-spectral resolution, imaging spectrometer operating in the solar reflective spectral range. One important capability of MERIS is providing full resolution data at $300 \mathrm{~m}$ resolution.
Figure 2 presents the results obtained by the multitemporal MODIS imagery of the volcanic plume evolution during October and November 2011 due to underwater volcanic activities. In this context, we have implemented a methodology for providing multitemporal/multisensor imagery information. Multisensorial satellite imagery obtained from MODIS and MERIS sensors have been processed to monitor the volcano activity and to provide information on the concentration of biological, chemical and physical marine parameters. Specifically, low resolution satellite estimations of optimal diffuse attenuation coefficient (Kd) and Chlorophyll-a (Chl-a) concentration under these abnormal conditions have been assessed.
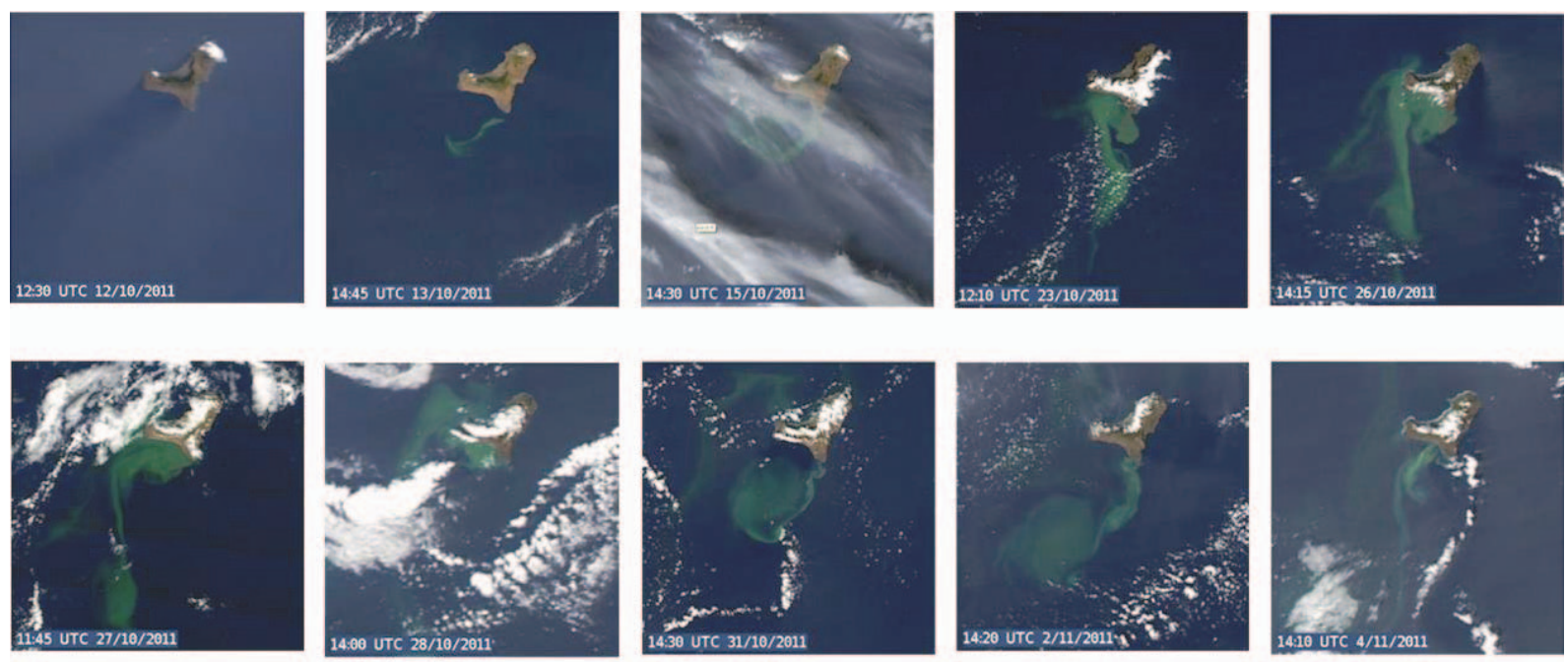

Figure 2. NASA MODIS RGB multitemporal images monitoring El Hierro submarine volcano. 


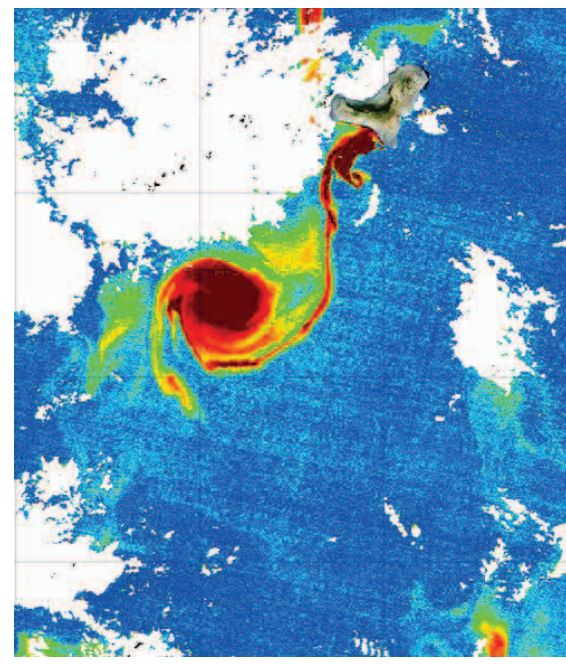

(a)

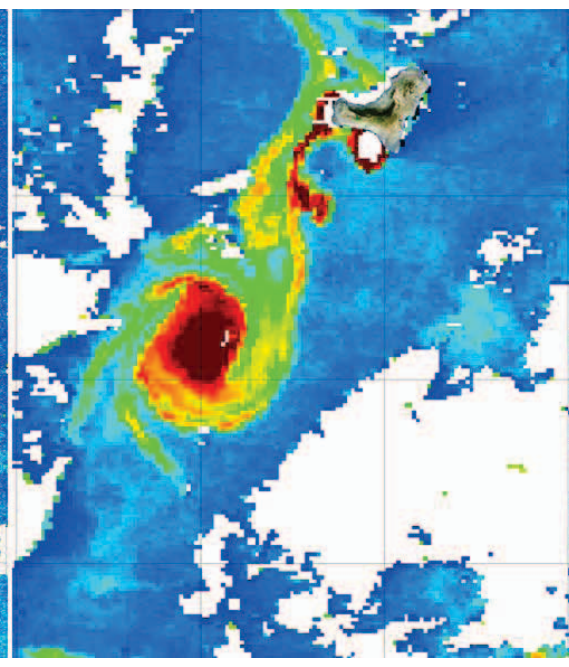

(b)

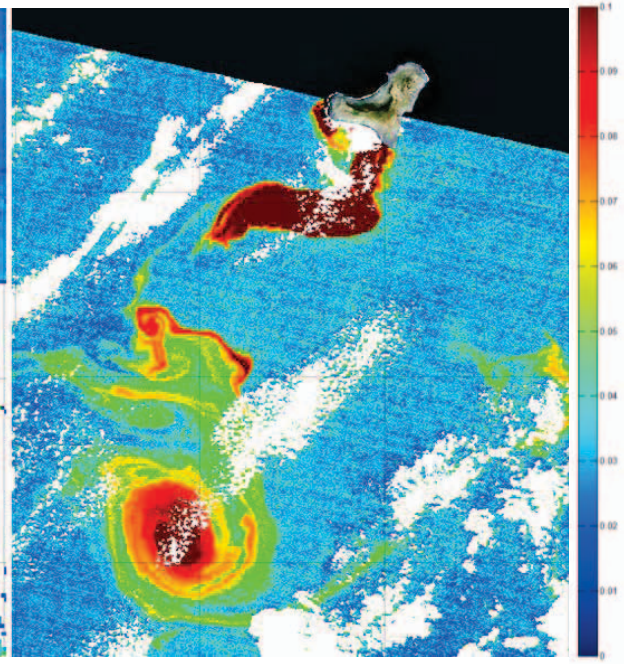

(c)

Figure 3. Images (Kd) acquired over El Hierro submarine volcano area in 2011: (a) MERIS on November 4, (b) MODIS on November 5 and (c) MERIS on November 9.

Chlorophyll-a concentration can be properly estimated from remotely sensed data in open ocean waters but it has been demonstrated a severe degradation in accuracy during the submarine eruption because the specific conditions, the alterations provoked in the water composition and the considerable turbidity [3]. On the other hand, the diffuse attenuation coefficient for downwelling irradiance at $490 \mathrm{~nm}$ (Kd) is more precise, thus we have selected this water turbidity indicator for the detection of the oceanographic structure. Figure 3 shows the evolution of an eddy using the $\mathrm{Kd}$ parameter from MERIS and MODIS images acquired on November 2011. It can be appreciated the eddy's displacement and rotation, as well as the presence of emerging filaments.

The precise submesoscale structure detection module is presented in Figure 4. The methodology is composed by two steps $[4,5]$ : (i) Initial structure detection: The first step is a pre-processing. Next, the initial segmentation module is applied. Several algorithms are incorporated in the module (Automatic and Manual Thresholding, K-means and Fuzzy K-means clustering, Expectation-Maximization clustering, etc.) to allow the maximum flexibility. Finally, a postprocessing step is required to remove isolated noisy pixels or to fill holes in the eddy structure due to clouds. (ii) Structure Growing: After the initial detection, the structure is well identified; however the fine detail of the emerging filaments cannot be well preserved. The precise delimitation is of fundamental importance when analyzing the structure and its evolution, so controlled growing techniques are applied only where these filaments appear. Points to grow and their directions are found using the skeleton of the initial structure detected. At each point, a window with the corresponding orientation and size masks the pre-processed image and new pixels are added to the structure after the application of thresholding or watershed region growing algorithms. This process is iteratively applied to achieve the desired segmentation. Figure 5 shows different example of the submesoscale front-like structure detected in the MERIS and MODIS images from Figure 3. Inner and outer contours are presented but the methodology is flexible to accommodate the needs of the oceanographic user.

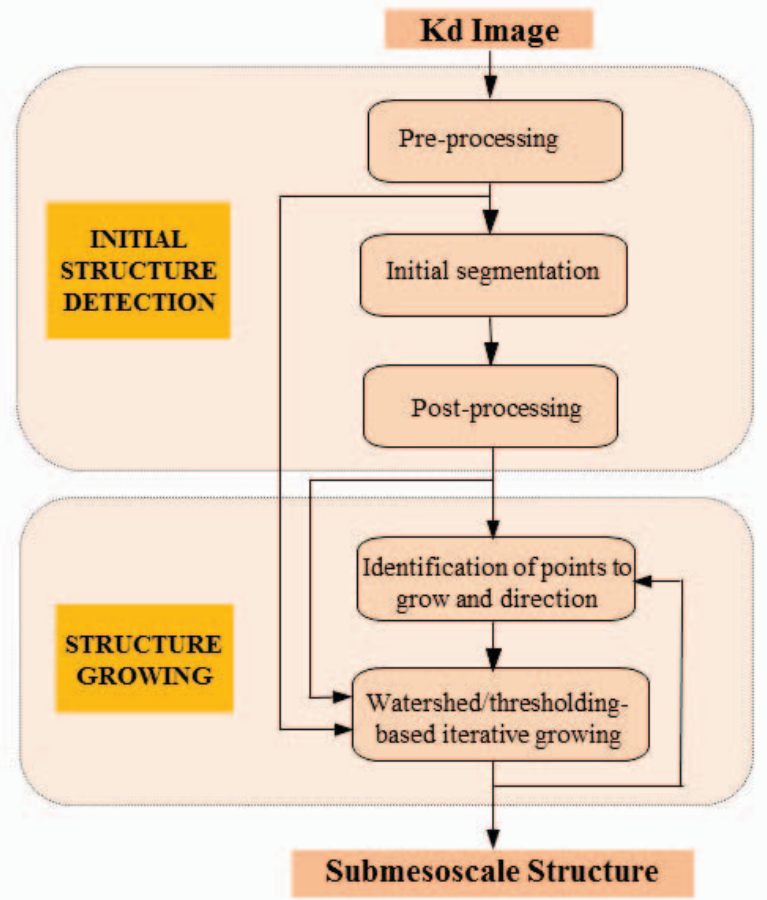

Figure 4. Methodology for the automatic segmentation of submesoscalar structures. 


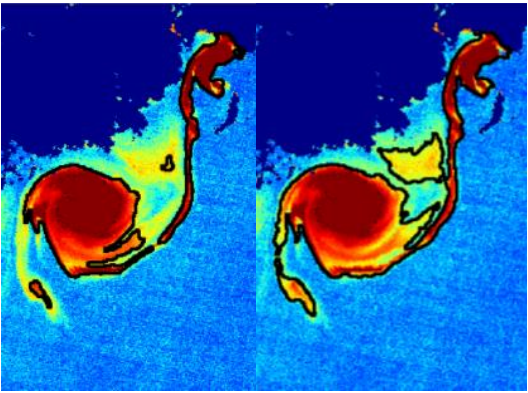

(a)

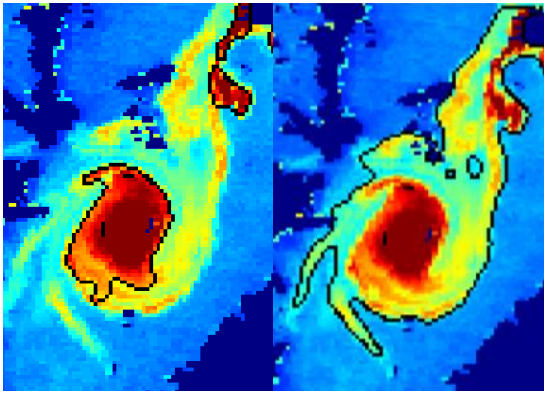

(b)

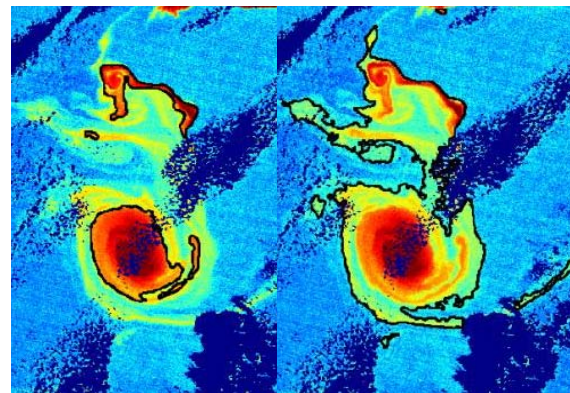

(c)

Figure 5. Inner and outer contours of the eddy and its filaments overlaid on the Kd image: (a) MERIS on November 4, 2011, (b) MODIS on November 5, 2011 and (c) MERIS on November 9, 2011.

\section{CONCLUSIONS}

The use of remote sensing for monitoring natural hazards has diversified in recent years owing to an increase in data availability and technological advances in their interpretation. Satellite remote sensing systems have proven useful for a range of applications including the capabilities to improve the understanding of submarine volcanic processes by analyzing remote sensing images providing more frequent observations and scientific information.

This paper describes an effort to integrate space-borne sensing data from MODIS (Terra and Aqua) and MERIS to improve global volcano monitoring and to provide information on submesoscale structures. In this context, the diffuse attenuation coefficient has been used and a two-step methodology has been developed. It is composed by initial structure detection and structure growing modules. The developed methodology obtains the initial coarse structure segmentation and achieves the maximum detail of the structure, especially for the anticyclonic eddy, after the iterative identification of the points to grow and the subsequent application of growing strategies. This detection approach has been validated over a database of MERIS and MODIS oceanographic products and it has demonstrated an excellent performance and robustness to noise and weak gradients, overcoming the problems associated with the use of edge detection techniques.

The favorable results have spawned a follow-up project that consists on the upgrade of the developed oceanographic techniques to study detailed the submesoscale structures and their possible relationship with lateral mixing and biological effects, combining low, medium and high-resolution multisensor satellite images of El Hierro tracer with in-situ observations, and implementing a surface motion model using optical flow algorithms applied in satellite image sequences.

\section{Acknowledgements}

This work has been supported by the PUMP Project (CTM2012-33355). MERIS data used here was supplied by ESA under Project 10134. We also would like to express our gratitude to NASA (OceanColor) for the provision of the MODIS data.

\section{REFERENCES}

[1] E. Fraile-Nuez, et al., "The submarine volcano eruption at the island of El Hierro: physical-chemical perturbation and biological response," Scientific Reports 2, doi:10.1038/srep00486, 2012.

[2] K. Joyce, S. Belliss, S. Samsonov, S. McNeill, S and P. Glassey, "A review of the status of satellite remote sensing and image processing techniques for mapping natural hazards and disasters," Progress in Physical Geography, 33, 2, 183-207, 2009.

[3] F. Eugenio, J. Martin, J. Marcello, and E. Fraile-Nuez, "Environmental monitoring of El Hierro Island submarine volcano, by combining low and high resolution satellite imagery," International Journal of Applied Earth Observation and Geoinformation, 29, 53-66, 2014.

[4] J. Marcello, F. Marqués and F. Eugenio, "Automatic tool for the precise detection of upwelling and filaments in remote sensing imagery," IEEE Transaction on Geoscience and Remote Sensing, 43, 7, pp: 1605- 1616, 2005.

[5] J. Marcello, F. Eugenio and F. Marqués, "Precise upwelling and filaments automatic extraction from multisensorial imagery," IEEE International Geosciences and Remote Sensing Symposium, Alaska, 2004. 